\title{
Magnetic resonance analysis of carbon content in paper mill sludge-soil mixtures used in remediation practices
}

\author{
A. Bonoli $\cdot$ V. Bortolotti $\cdot$ A. Dall'Ara $\cdot$ \\ P. Macini • M. Vannini
}

Received: 19 April 2013/Revised: 16 September 2013/Accepted: 5 November 2013/Published online: 28 November 2013

(C) Islamic Azad University (IAU) 2013

\begin{abstract}
One of the most promising recycling procedures of some industrial organic wastes, as paper mill sludge (PMS), is soil amendment. In this field of application, PMS and natural soil are mixed together according to specific regulations to avoid potential environmental risks that can arise after land restoration, and hence, the mixture must be scrupulously characterized and evaluated, also by means of different analytical techniques. Proton nuclear magnetic resonance $\left({ }^{1} \mathrm{H} \mathrm{NMR}\right)$ is a powerful, noninvasive and nondestructive technique to investigate natural and artificial porous media, and it is a promising tool to characterize amended soils as well, also in the light of the experimental difficulties that these materials generally entail. In this work, NMR micro- and macro-porosity were related to total organic carbon (TOC) content, and information on the behavior of organic matter $(\mathrm{OM})$ contained inside the mineral matrix was obtained. In particular, a high presence of TOC shows that micropores are forbidden to the saturating water, giving evidence of $\mathrm{OM}$ interactions with soil microstructure. This kind of analysis has clear
\end{abstract}

A. Bonoli · V. Bortolotti · P. Macini · M. Vannini ( $\)$

DICAM, Department of Civil, Chemical, Environmental and Materials Engineering, University of Bologna, 28 Via Terracini, Bologna, Italy

e-mail: marianna.vannini2@unibo.it

A. Dall'Ara

Material Technologies Technical Unit Faenza, ENEA, 186 Via Ravegnana, Faenza, Italy

M. Vannini

Operative Unit "Industrial Ecodesign, Waste Recycle and Life Cycle of Products", Interdepartmental Centres of Industrial Research "Energy and Environment", 22 Via Angherá, Rimini, Italy implications in the characterization of amended soil biological processes, such as biodegradation.

Keywords Natural soils $\cdot$ Paper mill sludge $\cdot$ Proton nuclear magnetic resonance $\cdot$ Total organic carbon

\section{Introduction}

Paper is one of the most widely recycled materials, and its manufacturing process produces many types of waste that can be eventually used in different recycling options. In particular, paper mill sludge (PMS) is the result of a paper cleaning-up process consisting of removing ink, fillers, coatings and contaminants from waste paper to recover reusable cellulose fibers (Usherson 1992). PMS types can be significantly different from a chemical standpoint, being strongly depending on the nature of the fibrous material, the chemical treatment of the manufacturing processes and the type of waste-handling practices adopted in the mill. Their nature is strictly associated with the type of waste paper (Jackson and Line 1997). In Italy, as well as in Europe, PMS is less and less considered as a waste (and, as such, disposed of to landfill or incinerated), and the reuse for environmental remediation (such as landscaping and dismissed quarry or mining areas reclamation) is strongly recommended due to its stability in aerobic conditions (Assocarta 2012). It is worthy to recall that, thanks to the presence of cellulose and wood fibers, PMS is a biomass rich in organic content, and therefore, anaerobic degradation (with $\mathrm{CH}_{4}$ and $\mathrm{CO}_{2}$ production) is very likely to happen. Because of this, PMS must be mixed with inactive soil in established percentages (MD, Italy, 1998) to prevent biogas production, which can cause severe environmental problems or even endanger nearby facilities or 
communities (Bonoli and Dall'Ara 2012). To avoid environmental risks, the composition of PMS and soil mixture (hereinafter PMSSM, Paper Mill Sludge and Soil Mixture) must be thoroughly evaluated. A classic approach in soil sciences is to measure the chemical total organic carbon content (TOC) and the total organic carbon-to-nitrogen ratio $(\mathrm{TOC} / \mathrm{N})$. This ratio is an important indicator of natural soil conditions, since its constancy is a distinctive characteristic of the quality of soils, sediments and sedimentary rocks, and by extension of PMSSM. In natural soils, TOC/N generally ranges from 10 to 12 and often decreases with depth, in some cases attaining values $<5$. In general, favorable conditions for the decomposition of organic residues narrow the range of TOC/N values (Stevenson and Stevenson 1994). Likewise, TOC/N is used to estimate the risks associated with this recycling procedure, since a specific TOC/N threshold value establishes the risk of biogas production in polluted soils (Adani 2004). TOC/ $\mathrm{N}$ ratio, together with $\mathrm{P}, \mathrm{Ca}, \mathrm{Mg}, \mathrm{Na}$ and $\mathrm{K}$ concentrations can be used to better characterize PMSSM. In poor organic matter (OM) soils, PMS are land spread to improve soil properties since their TOC content, due to fibers of cellulose, is about $45-85 \%$ (Beauchamp et al. 2002). The use of PMS, or raw deinking sludge in general, as a soil remediation amendment, is potentially limited by the possible lack of $\mathrm{N}$ and $\mathrm{P}$ for adequate plant nutrition, and so if $\mathrm{N}$ and $\mathrm{P}$ fertilizers are added, PMS can be effectively used (Fierro et al. 1997). Furthermore, it has been observed that the quantity of $\mathrm{OM}$ can influence soil texture, and the relationship between the soil OM content and the volume of different pore size fractions has been studied. In particular, this is especially true for micropores, in fact an increase in TOC content is observed in fine-sized aggregates (Kirchmann and Gerzabek 1999).

The increasing use of recycled materials in soil remediation activities involves environmental implications not yet well defined, which are objects of study of numerous and interdisciplinary research fields. Possible contamination concerns stimulated the use and customization of innovative techniques to characterize these materials. In this context, proton nuclear magnetic resonance $\left({ }^{1} \mathrm{H}\right.$ NMR) is a powerful noninvasive and nondestructive technique that can be adequately employed to investigate porous media in the time domain (Time Domain NMR, TDNMR). This is true not only for consolidated porous media (e.g., rocks), but also for unconsolidated ones (e.g., soils), so the idea was to apply TD-NMR to characterize natural soils amended with recycled materials, such as PMS.

TD-NMR studies the interactions of porous media saturated with liquids containing hydrogen nuclei, namely water or oil. Fluids with ${ }^{1} \mathrm{H}$ nuclei confined in porous media are used to look into the structural properties of high surface-to-volume ratio systems (such as rocks and soils). The ${ }^{1} \mathrm{H}$ TD-NMR signal is proportional to the total amount of water inside the pore space and is generally related to the proton relaxation, by means of the analysis of the longitudinal $\left(T_{1}\right)$ and transverse $\left(T_{2}\right)$ relaxation times. There is a correspondence between the relaxation times distribution and the micro-geometric features of these systems. In porous rocks, the values of $T_{1}$ and $T_{2}$ are affected by many factors, such as the physical and chemical properties of the saturating fluids, the uniformity of the static magnetic field, the variations in pore sizes, the differences between the magnetic susceptibility of pore fluids and rock grains, the fluid viscosity and diffusion and the presence of paramagnetic ions on the pore walls or dissolved in fluids (Dunn et al. 2002). Application of TD-NMR techniques in petrophysical and geophysical sciences are today well established; however, it is not yet frequently used in soil science, although it exhibits great potential. Heterogeneity of soils and the particular structure of OM contained in soils (not yet fully understood) do not allow one to easily transfer one-to-one TD-NMR geophysical or petrophysical studies to soil science (Bayer et al. 2010). Moreover, in natural soils, the paramagnetic ions concentration is high enough to significantly change the surface relaxivity $\rho$ (the NMR relaxation strength due to the interactions of ${ }^{1} \mathrm{H}$ nuclei with pore surface), by increasing $\rho$ of some order of magnitude if compared with systems with no paramagnetic ions. Due to the mixing with waste materials, PMSSM often contain a higher concentration of paramagnetic ions with respect to natural soils, making the application of TD-NMR techniques more difficult.

The purpose of this study is to investigate the use of TD-NMR techniques to characterize PMS used for soil reclamation of a dismissed quarry area, in order to get information on how the high content of OM can affect the distribution of relaxation times. In particular, the correlation of $T_{1}$ relaxation time distributions $\left(T_{1}\right.$ distributions for short) with respect to TOC content has been investigated. As mentioned above, PMSSM often pose several experimental problems, and meticulous experimental care must be adopted. In fact, the presence of paramagnetic ions significantly influences data measured in the time domain, possibly inducing substantial misinterpretation of the inverted relaxation time distribution. In fact, the inversion of multiexponential relaxation data is a notoriously ill-posed problem (Provencher 1982), and thus, particular care must be adopted to prevent the elaboration of relaxation time distributions with artifacts details which might be misinterpreted, for example, as physically meaningful resolved porous compartments.

It is important to recall that the particular sample preparation procedure (quantity of saturating water, mixing 
and compaction procedure, etc.) affects the pore distributions, and so the shape and modality of relaxation time distributions. Thus, particular care has been used to prepare and investigate the samples. Moreover, the $T_{2}$ analysis usually adopted in soil science, normally performed with the standard Carr-Purcell-Meiboom-Gill sequence (Bayer et al. 2010), cannot easily be used in quantitative analyses of PMSSM samples, because of their strong paramagnetic ions content.

Regarding the experimental apparatus, it is worth recalling that the nature of PMSSM samples and the large dimensions of the dedicated probe used (to accommodate a more representative quantity of PMSSM) forced the NMR relaxometer to take measurements close to its instrumental limits so that in some cases, its electronic dead time was comparable with the length of the free induction decay (FID) signal to be acquired, excluding therefore the possibility to use, for example, the standard inversion recovery sequence. To overcome all these problems, it has been necessary to develop a more convenient NMR sequence to obtain more reliable information from NMR signal. PMSSM samples have been characterized by $T_{1}$ relaxation times analysis, using the specifically developed sequence (the logarithmically distributed aperiodic saturation recovery spin echo sequence, LAPSRSE) that overcomes many measurement difficulties (Vannini et al. 2012). The $T_{1}$ analysis shows that a correlation between the percentage of PMSSM micropores and TOC content exists, that is, the higher the TOC, the lower the presence of water in the micropores. TD-NMR has shown to be a valuable tool to characterize PMS biological features and its evolution over time. It can check the correct range of soil TOC content and easily monitor biological biodegradation of soils.

\section{Materials and methods}

\section{Soil samples}

All analyzed soil samples come from a land reclamation area (formerly a serpentine quarry site) located in Northern Italy, in which authorized (EWC 2002) wastes were used to reclaim the hillside profile. The volume of the authorized wastes was about $12,000 \mathrm{~m}^{3}$. The authorized wastes in the soil mixture are the following: foundry waste materials (EWC code 100202, 100903 furnace slag); soil and stones not containing dangerous substances (EWC code 170504) and PMS (EWC code 030305 de-inking sludges from paper recycling and 030310 fiber rejects; fiber-, filler- and coating-sludge from mechanical separation). PMS is about onethird of the total authorized waste volume. Both 030305 and 030310 PMS come from paper mills that produce paper waste (chlorine free) and generally contains cellulose fibers too small to be reused, together with mineral charges and residual inks. The use of PMS for land reclamation was authorized to prepare PMSSM with a PMS percentage not higher than $30 \%(\mathrm{w} / \mathrm{w})$, calculated for a PMS with $27 \%$ minimum dry matter (MD, Italy, 1998). In order to characterize the PMSSM, which had to comply with specific European and Italian regulations (MD, Italy, 1998), five samples have been selected, whose TOC content ranges from that of natural soil $(<1 \% \mathrm{w} / \mathrm{w})$ up to $20 \% \mathrm{w} / \mathrm{w}$ and more, in order to cover a wide range of possible TOC values.

\section{TOC and $\mathrm{N}$ analysis}

The determination of the TOC and $\mathrm{N}$ have been obtained by quantitative elemental analysis, a process where a sample of solid material (e.g., soil, waste, sludge) is analyzed on the basis of its elemental composition, according to Dumas method, included in official methods for soil analysis (MD, Italy, 1999). Major constituents of organic substances are $\mathrm{C}, \mathrm{H}, \mathrm{N}, \mathrm{S}$ and $\mathrm{O}$, beside halogens, $\mathrm{P}$ and metals. In this case, the aim was to determine the elemental composition of PMSSM, in particular the $\mathrm{CO}_{2}$ and $\mathrm{N}_{2}$ percentage. The Dumas method is based on a complete and instantaneous oxidation (flash combustion) with subsequent conversion of all organic and inorganic substances into gases. They are convoyed, in helium stream, on an appropriate catalyst layer to complete the oxidation process and then on copper layer, in order to reduce $\mathrm{N}$ oxides in $\mathrm{N}_{2}$. TOC analyses were performed by means of infrared detection of $\mathrm{CO}_{2}$ using a LECO RC-412 (Leco Inc., St. Joseph, MI, USA); N concentration was determined by means of a thermo-conductivity detector after gas-chromatographic separation with a Thermo Flash EA 1112 (Thermo Fisher Scientific Inc., Boston, USA). Both apparatuses are located at ARPA, the Italian National Agency for Environmental Protection, Emilia Romagna Laboratories (Provincial Section, Ravenna, Italy).

Samples preparation and NMR data acquisition

NMR tests were performed with a home-made full-size NMR relaxometer composed of a console Stelar (Stelar Srl, Mede, Italy) and a $0.2 \mathrm{~T}$ low field permanent magnet corresponding to $8 \mathrm{MHz}$ for protons, part of the ARTOSCAN $^{\mathrm{TM}}$ tomograph (ESAOTE SpA., Genova, Italy) located at the LAGIRN labs of the University of Bologna. The relaxometer houses probes of different size, up to $13 \mathrm{~cm}$ in diameter. The dedicated NMR probe utilized for data acquisition was equipped with a transmit-receive coil having a sensitive volume of about $16 \mathrm{~cm}^{3}$ (diameter 
$26 \mathrm{~mm}$, length $30 \mathrm{~mm}$ ) specifically adapted for these experiments. This large coil probe permits to measure a significant quantity of sample, but, at the same time, it implies a long dead time (about $<25 \mu$ s). Since sample preparation can strongly affect the NMR results, particular care was adopted at this stage. Before NMR experiments, all PMSSM were dried at $40{ }^{\circ} \mathrm{C}$ (a low temperature to minimize OM damage) for $24 \mathrm{~h}$ in a ventilated oven and sieved (ASTM mesh 10, ASTM International, http://www. astm.org). The overflow was discarded, and only the underflow was utilized in lab investigations. The underflow (a dry powder) was rehydrated with a fixed proportion of water (40\% of dry mass) and mixed apart, then placed in cylindrical test tubes (diameter $25 \mathrm{~mm}$ ), which were filled to approximately $20 \mathrm{~mm}$ for compatibility with NMR coil dimensions (to fit into a more favorable volume of magnetic field homogeneity). The material was compacted inside the test tubes according to a standard procedure devised for this type of experiment. The headspace above the mixture surface, with respect to the sensitive height of the coil (about $10 \mathrm{~mm}$ ), allows for possible swelling of the mixture. Test tubes were sealed with Teflon tape and wrapped at the top with Parafilm to minimize evaporation.

The spin-lattice relaxation data, necessary to compute the $T_{1}$ distributions, are usually acquired using the traditional inversion recovery (IR) sequence. IR can be very time consuming: it requires the return to the thermal equilibrium of the magnetization before each repeated scan, and it does not work properly in situations in which severe instrumental imperfections combines with sample complexity. Moreover, the duration of the inversion RF pulse, the so called $180^{\circ}$ pulse, used by above relaxometer is comparable with the duration of the FID signal. In fact, the presence of intense paramagnetic impurities destroys the FID signal in the order of few tens of microseconds, and as a result, the efficiency of the IR inversion is very poor, $<40 \%$. A possible alternative is to use saturation recovery (SR), which is faster and does not need any inversion pulse (Blümich 2003). As we chose to operate without using gradient spoiler pulses (to reduce the complexity of the hardware, and of the tuning and set up procedures), to enable SR to work properly, it is necessary to use a suitable magnetization suppression pulse sequence, in which a burst of non-selective pulses reduces the effective magnetization to zero. Therefore, we did not try to obtain $S / V$ and $\rho$ by means of the time dependent diffusion coefficient in restricted geometry (Hürlimann et al. 1994). The use of the LAPSRSE, which is an extension of the well-tested sequence logarithmically distributed a-periodic saturation recovery (LAPSR-Gombia et al. 2008) permits to obtain the spin-lattice relaxation data, by means of a sequence of pulses to suppress the magnetization of the sample and allows a fast and efficient pre-saturation in the presence of a wide spread of offsets, relaxation rates, and magnetic field imperfections. To overcome the dead time limitation, which for many PMSSM samples is comparable with the FID duration, it has been necessary to adopt an echo signal detection method. In practice, the result of a $T_{1}$ experiment is a collection of digitized and recorded echoes signals. Therefore, the LAPSRSE data file consists of an header, with all the experiment parameters, followed by a set of data blocks each of which represents a recorded echo signal. For this multi-block sequence, 64 echoes were acquired from an initial LAPSRSE saturation recovery time of $50 \mu \mathrm{s}$ to a final time equal to four times the maximum $T_{1}$ value expected. This parameter ranged between 40 and $100 \mathrm{~ms}$ according to the maximum $T_{1}$ value of the PMSSM samples. In all experiments, in order to be sure that all the transversal magnetization components were destroyed before starting the real acquisition, the time between the last pulse of the saturation pulses preamble and the first excitation pulse was set on the order of $100 \mu \mathrm{s}$. Also the echo time was the same for all experiments and, as the dead time was about $25 \mu \mathrm{s}$, it was fixed at $60 \mu \mathrm{s}$. The duration of the $90^{\circ}$ excitation pulse was $11 \mu \mathrm{s}$. Every sequence was repeated for 32 scans to obtain an acceptable signal to noise ratio, which was on average approximately of 250 .

In order to obtain quasi-continuous relaxation time distributions, all data have been processed by means of the software UpenWin (Bortolotti et al. 2009), a Windows program specifically designed not to give distribution details that are not supported by data. The smoothing method used by the software can give sharp distribution lines, not broadened more than it is consistent with the data noise. Thus, the resulting distributions do not have several peaks not required by the data to be separate, helping to prevent a physical misinterpretation of data.

$\mathrm{T}_{1}$ cut-off determination

In soil science literature, there is no univocal definition of the terms micropores, mesopores and macropores, probably due to the large number of different techniques used to determine the pore size distributions (Zdravkov et al. 2007). This ambiguity suggested the use of the typical petrophysical cut-off method to identify micropores by NMR. The portion of capillary-bound water in the pore space, called irreducible water saturation, is related to the short components of the NMR relaxation times and for an estimate it can be useful to set a specific relaxation time (cut-off) below which the water is immobile (Dunn et al. 2002). Using the cut-off method, usually based on a fixed $T_{2}$, that divides the $T_{2}$ distribution into two parts, the 
irreducible water can be estimated. The two parts are related to pores containing bound water and pores containing free water (which we identified as water in macropores), respectively. In this study, the cut-off is applied to $T_{1}$ distributions, and the desaturation was obtained with the centrifuge technique, a common approach in petrophysical applications (Coates et al. 1999). When the mass of the centrifuged sample becomes constant, it is supposed that the respective $T_{1}$ distribution is indicative of the distribution of irreducible water saturation. Therefore, it is possible to deduce a $T_{1}$ cut-off value below which the $T_{1}$ values represent only bound water, which we assumed mainly as the water filling the micropores. PMSSM samples used for cut-off experiments were prepared following the same procedure described above, and NMR measurements were performed after each centrifuge run, to follow the decrease in water mass and to use the total NMR signal detected to test the ability of the NMR apparatus to correctly detect the mass of water loss.

\section{Relaxivity evaluation}

In this study, we supposed that saturated samples are in the fast diffusion regime, since fluids are confined in a porous medium. Under this hypothesis the longitudinal relaxation time of confined ${ }^{1} \mathrm{H}$ fluids is related to the surface relaxivity coefficient $\rho$ and to the $S / V$ ratio for each pore. Almost all MRR studies on these kinds of samples assume that spins system satisfy this condition. Tests to evaluate the dependence of the $T_{1}$ distribution with respect to the temperature can be made. In fact, if a sample is not in the fast diffusion regime, then the relaxation time of confined fluid is related to $D$, the diffusion coefficient of spins in the pore fluid, and $D$ is very sensitive to the temperature variations. Conversely, $\rho$ for many kinds of samples is not appreciably sensitive to temperature variations. So, a null or a weakly dependence of the $T_{1}$ distribution with respect to the temperature variations could be assumed, in a first rough approximation, as an indication that the sample is in the fast diffusion regime (Dunn et al. 2002). Tests were made on $T_{1}$ distributions of a single PMSSM sample measured at three different temperatures, 5,20 and $40{ }^{\circ} \mathrm{C}$, respectively, which proved that samples are in the fast diffusion regime. In fast diffusion, three independent relaxation mechanisms, acting in parallel, are involved: bulk fluid processes and surface relaxation, which affect both $T_{1}$ and $T_{2}$ relaxation, and the diffusion in the presence of magnetic field gradients, which only affects $T_{2}$ relaxation. Moreover, the geometric information of the porous system is only characterized by the internal pore surface area to pore volume ratio $S / V$ (Dunn et al. 2002). So that, for $T_{1}$ :
$\frac{1}{T_{1}}=\frac{1}{T_{1 \text { bulk }}}+\frac{1}{T_{1 \text { surface }}}=\frac{1}{T_{1 \text { bulk }}}+\rho_{1} \frac{S}{V}$,

where $\rho_{1}$ is the longitudinal surface relaxivity. The presence of para and ferromagnetic ions on the pore surface causes strong surface relaxation. The strength of the surface effect is usually represented by the velocity at which non-equilibrium nuclear magnetization flows out of the pore fluid at the surfaces, giving an increase $\rho_{1} S / V$ in the relaxation rate of the fluid in a pore if diffusion is sufficient to maintain the nuclear magnetization nearly constant over the pore. In order to use Eq. (1) to evaluate a pore size distribution from a relaxation time distribution, supposing bulk relaxation effects negligible, it is necessary to know the value of $\rho_{1}$ and suppose that it is roughly the same over the whole sample. The same Eq. (1) can be used to evaluate $\rho_{1}$, but in this case one needs the measurement of $S / V$, which in general it is not easy to measure, and whose result could change significantly depending on the measurement method adopted (Kleinberg 1999). Looking for a correlation between relaxation data and a single parameter, such as $S / V$, it is necessary to reduce the relaxation data to a single parameter. A large number of different such single "effective" relaxation times can be formed from the relaxation distribution. In particular immediately after an abrupt change in non-equilibrium magnetization at $t=0$ (for instance, by a $90^{\circ}$ pulse) the magnetization is uniform over the pore space even in slow diffusion regime. Therefore, if $\rho_{1}$ is constant over $S$, the instantaneous relaxation rate at $t=0$ should be $\rho_{1} S / V$, whatever the diffusion regime or pore size distribution. This initial relaxation rate $R_{1}(0)=1 / T_{1}(0)$, is the arithmetic average rate if a distribution of rates exists so that:

$\frac{S}{V}=\frac{R_{1}(0)}{\rho_{1}}$

with

$R_{1}(0)=-\left.\frac{d}{d t} \ln (S(t))\right|_{t=0}=-\left.\frac{d S(t)}{d t} \frac{1}{S(t)}\right|_{t=0}$

where $S(t)$ is the reversed longitudinal relaxing signal (extrapolated initial value of each LAPSRSE data blocks minus the extrapolated initial value of the block recorded at long inversion time, theoretically the echo signal recorded at infinite time. $R_{1}(0)$ extrapolates both the signal and the slope of the $T_{1}$ relaxation curve back to an LAPSRSE saturation recovery time $t=0$ (Borgia et al. 1996).

In case of non-consolidated materials, constituted by grains approximately spherical of diameter $d$ and randomly packed, $S / V$ can be estimated by the following relationship: 
$S / V=6(1 / \varphi-1) d^{-1}$,

where $\varphi$ is the porosity (Vogt et al. 2002). In this case, substituting the above relationship in Eq. (1) gives:

$R_{1}(0)=R_{1 b}+\rho_{1} 6\left(\frac{1}{\varphi}-1\right) d^{-1}$,

$R_{1}(0)$ is not the only possible NMR parameter useful for the determination of $\rho_{1}$. Various single parameters (or kinds of averages) can be computed from distributions, as the relaxation parameters $T_{1}$ peak of the distribution and the geometric weighted average time $T_{1}\left(T_{1 \mathrm{G}}\right)$. It is remarkable that $R_{1}(0)$ is computed directly on measured data and not on the distribution relaxation time; thus, it completely overcomes the troubles of ill-posed problems. It is worth emphasizing that the presence of paramagnetic sites increases the relaxation of nuclear spins in an otherwise diamagnetic bulk environment. Hence, provided that the concentration of dissolved paramagnetic ions is not too high, $R_{1 \mathrm{~b}}$ is a sum of the paramagnetic and diamagnetic contributions (Mitreiter et al. 2010). So $R_{1 \mathrm{~b}}$ can be written as a sum of $R_{1 \mathrm{~b} 0}$, which refers to the relaxation rates in absence of paramagnetic species and the product of the concentration $[M]$ of paramagnetic substances with $r_{i}$ $\left(\mathrm{mM}^{-1} \mathrm{~s}^{-1}\right)$ the enhancement of the longitudinal nuclear spin relaxation rates due to the presence of $M$ (Helm 2006).

In order to estimate $\rho_{1}$, using Eq. (5), the PMSSM sample S2 was sub-sampled in five not overlapping granulometric classes, to avoid grains mixing (frequently, in soils the small grains remain attached to the large ones, forming aggregates) (Pignatello 1998). Sieves classes used were: $1,680-1,190,707-595,425-300,250-150$ and $125-63 \mu \mathrm{m}$. Five glass vials (diameter $8 \mathrm{~mm}$ ) were partially filled (height $25 \mathrm{~mm}$ ) following the sample preparation procedure reported above, and NMR measurements were performed. The minimum time of echo of the LAPSRSE should take in account the dead time, so it was set on the order of $50 \mu \mathrm{s}$. A value for $R_{1}(0)$ was obtained from each LAPSRSE measurement. Special care has been taken to minimize the scatter in the computed results. So, computations were made on sequences of stacks of data blocks (in particular each stack is averaged on 4 blocks) with the last stack subtracted from each of the others. The time of each stack was computed as the geometric mean of the times of the blocks composing the stack. Data elaboration has been performed using a home-made software, which can evaluate the $S(t)$ natural logarithm and its linear behavior (shown by Eq. 5) at the very short time of the trend, using the first 20 points. The porosity of sample S2 was estimated with a gas porosimeter. Then, an estimation of $\rho_{1}$ has been calculated by means of Eq. (5). It is important to highlight that a unique relaxivity value for all analyzed samples has been used, deduced by only one PMSSM measured sample, introducing a systematic error in the further calculation of the pore sizes distributions. Some Authors (Mikutta et al. 2004) indeed found that the mineral soil phase and the mineral soil phase contaminated with polygalacturonic acid have different values for relaxivity, because this acid significantly reduces the specific surface area (SSA) of the mineral phase. Furthermore, in soils the TOC is in general correlated with the SSA, so it is likely to expect that TOC may influence the relaxivity values.

An alternative method to evaluate the relaxivity (besides the above intrinsic NMR method) is through the measure of the SSA, and in this study it has been used as a comparative method. The $\mathrm{N}_{2}$ BET (Pennell et al. 1995) method has been adopted to estimate SSA. It is known that the cleaning procedure used in the BET method might alter the samples (Borgia et al. 1996), for this reason, to avoid OM damage, the cleaning procedure was performed at room temperature. Moreover, it has been observed that $\mathrm{N}_{2}$ molecules are subject to molecular sieving, and therefore, $\mathrm{N}_{2}$ BET is not a suitable adsorbate for materials having pores smaller than $0.5 \mathrm{~nm}$ (De Jonge and Mittelmeijer-Hazeleger 1996). So, as a general rule, it is necessary to take in account that, by using the BET method, the measured SSA can be one or two orders of magnitude smaller than the values obtained with other methods (Kleinberg 1999). Thus, the relaxivity has been estimated using Eq. (2), where now $\mathrm{S}$ is the BET specific surface $\left(\mu \mathrm{m} \mathrm{g}^{-1}\right), V$ the specific porosity volume of the sample $\left(\mathrm{m}^{3} \mathrm{~g}^{-1}\right)$ and $R_{1}(0)$ the usual average relaxation rate.

\section{Results and discussion}

\section{Chemical analysis}

Table 1 reports the results of the chemical analysis (TOC and $\mathrm{N}$ content, expressed in weight for weight percent, \% $\mathrm{w} / \mathrm{w}$ ), and the TOC/N of the five investigated samples. The

Table 1 Chemical analysis of the five investigated samples

\begin{tabular}{lllc}
\hline Sample & TOC $(\mathrm{w} / \mathrm{w} \%)$ & $\mathrm{N}(\mathrm{w} / \mathrm{w} \%)$ & $\mathrm{TOC} / \mathrm{N}$ \\
\hline S1 & 0.7 & 0.06 & 11.7 \\
S2 & 1.1 & 0.07 & 15.7 \\
S3 & 9.3 & 0.05 & 186 \\
S4 & 10.6 & 0.22 & 48.2 \\
S5 & 21.2 & 0.07 & 303
\end{tabular}

Total Organic Carbon (TOC), Nitrogen (N) and Total Organic Carbon-to-Nitrogen Ratio (TOC/N) are reported 
Fig. $1 T_{1}$ distributions of all five PMSSM samples at the end of the centrifuge procedure. The valley between the two populations varies between 10 and $20 \mathrm{~ms}$. The inset shows the $T_{1}$ distributions of PMSSM S5 during the centrifuge procedure

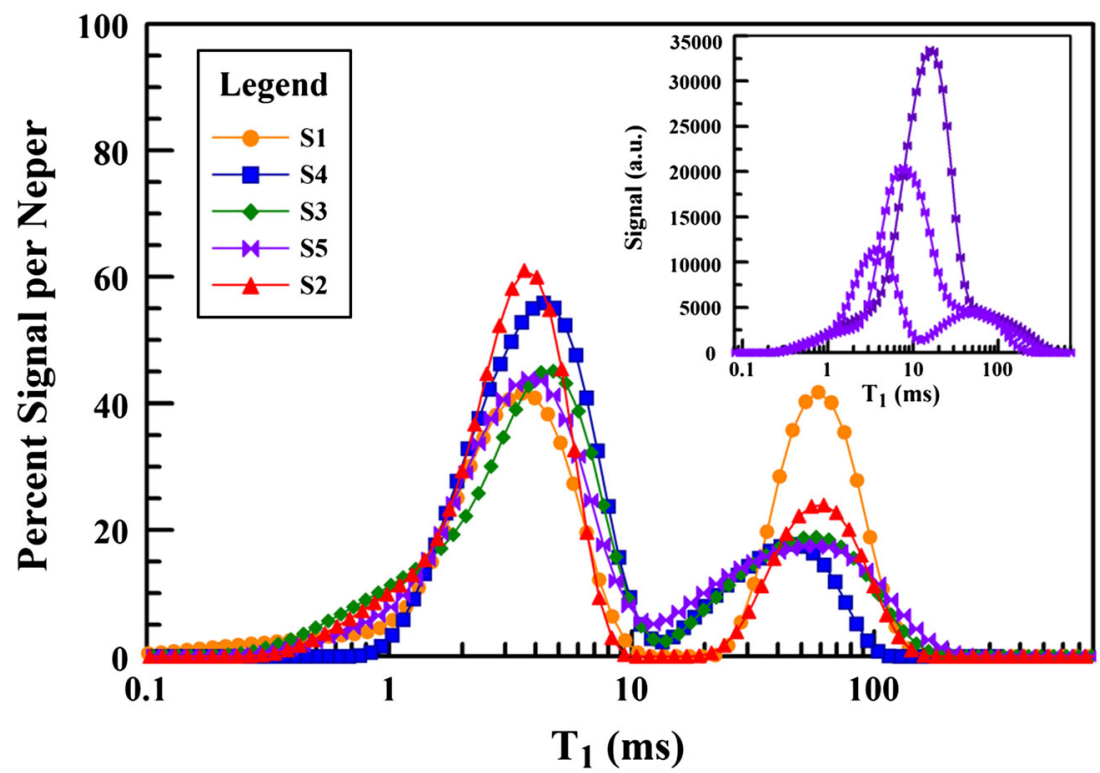

TOC/N ratio of original soils before remediation, evaluated in an area close to the coring site, is in the range of 10 on average (data not shown). The five samples show some differences. In particular, three samples (S3, S4 and S5) have a high TOC content, probably due to an in situ incorrect mixing of soil and PMS, with a disproportion in terms of PMS.

$\mathrm{T}_{1}$ distributions and $\mathrm{T}_{1}$ cut-off determination

$T_{1}$ distributions of PMSSM samples were measured after preparation and after each centrifuge run. $T_{1}$ distributions of sample measured immediately after preparation (data not shown) have a wide monomodal distributions, with small tails on both sides and a spread in a range of relaxation times between a few hundred of $\mu$ s and a few hundred ms. The $T_{1}$ distribution of a natural soil sample, cored nearby the reclamation site, is quite similar to the one of sample S2, indicating that this sample is similar in carbon and nitrogen content with respect to the natural soil of the nearby area. After every centrifuge run it is possible to track the changes of the $T_{1}$ distributions due to desaturation. An example is reported in the inset of Fig. 1, showing the variations of $T_{1}$ distribution of sample $\mathrm{S} 5$ before and after two centrifuge runs. A bimodal distribution appears during desaturation, suggesting the formation of two distinct populations, which in part can be explained observing that samples are unconsolidated and so, during centrifuge runs, a forced gravimetric redistribution of the grains might occur. The bimodal behavior is observed on all centrifuged samples (Fig. 1). It can be assumed that the peak at short times represents the irreducible water, and hence, its range of time is supposed to be the range of $T_{1}$ times of water in the micropores. In addition, it can be assumed that the valley between two peaks corresponds to the cut-off position useful to define the range of micropores. The cut-off is slightly different from sample to sample, so a specific $T_{1}$ cut-off for each sample has been evaluated by means of a minima study. Results are the following: sample $\mathrm{S} 1, T_{1 \text { cut- }}$ off $=20 \mathrm{~ms} ; \mathrm{S} 2,11 \mathrm{~ms} ; \mathrm{S} 3,14 \mathrm{~ms} ; \mathrm{S} 4,13 \mathrm{~ms} ; \mathrm{S} 5,12 \mathrm{~ms}$. $T_{1}$ distributions not only show the relaxation curve shift to low times but also, correctly, give an account of the quantity of water loss, used as a general quality test of the correctness of the particular measurement procedure implemented in this study. A good linear relation between the total NMR signal and the water loss during the desaturation of sample S5 was observed (data not shown).

\section{Relaxivity determination}

The $T_{1}$ relaxation time curves of the five sieved sub-samples are quite well-defined monomodal curves. Figure 2 shows the $R_{1}(0)$ of the five sub-samples plotted versus the inverse of the particles grain diameter, computed by means of Eq. (3) on a plot of $\ln S(t)$ versus $t$ (see the inset of Fig. 2). $\rho_{1}$ was estimated by means of Eq. (5), using a constant $\varphi=0.4$, and its value is about $180 \mu \mathrm{m} \mathrm{s}^{-1}$ on average. In general, this seems a high value for surface relaxivity, nonetheless values of this order of magnitude are reported in literature (Bryar et al. 2000). This relaxivity value has been compared with those obtained using the rate of $T_{1 \mathrm{G}} ; \rho_{1}$ calculated for $T_{1 \mathrm{G}}$ is around $330 \mu \mathrm{m} \mathrm{s}^{-1}$. The relaxivity value obtained from $T_{1 \mathrm{G}}$ is comparable with the one obtained in a previous work on similar PMSSM samples (Vannini et al. 2012). Using this value of $\rho_{1}$, according to $T_{1}$ distributions before the centrifuge procedure, an 
Fig. 2 Linear trend of $R_{1}(0)$ versus the inverse of grains mean diameters; by using Eq. (5) it is possible to deduce $\rho_{1}$ from the slope. The inset reports an example of a plot of the natural logarithm of the signal versus time, used to compute $R_{1}(0)$ of each sample; $\tau$ is the LAPSRSE inversion time. Only the first $10 \mathrm{~ms}$ have been considered in order to exploit the linear behavior

Fig. 3 Percentage of NMR signal of water in micropores versus TOC content
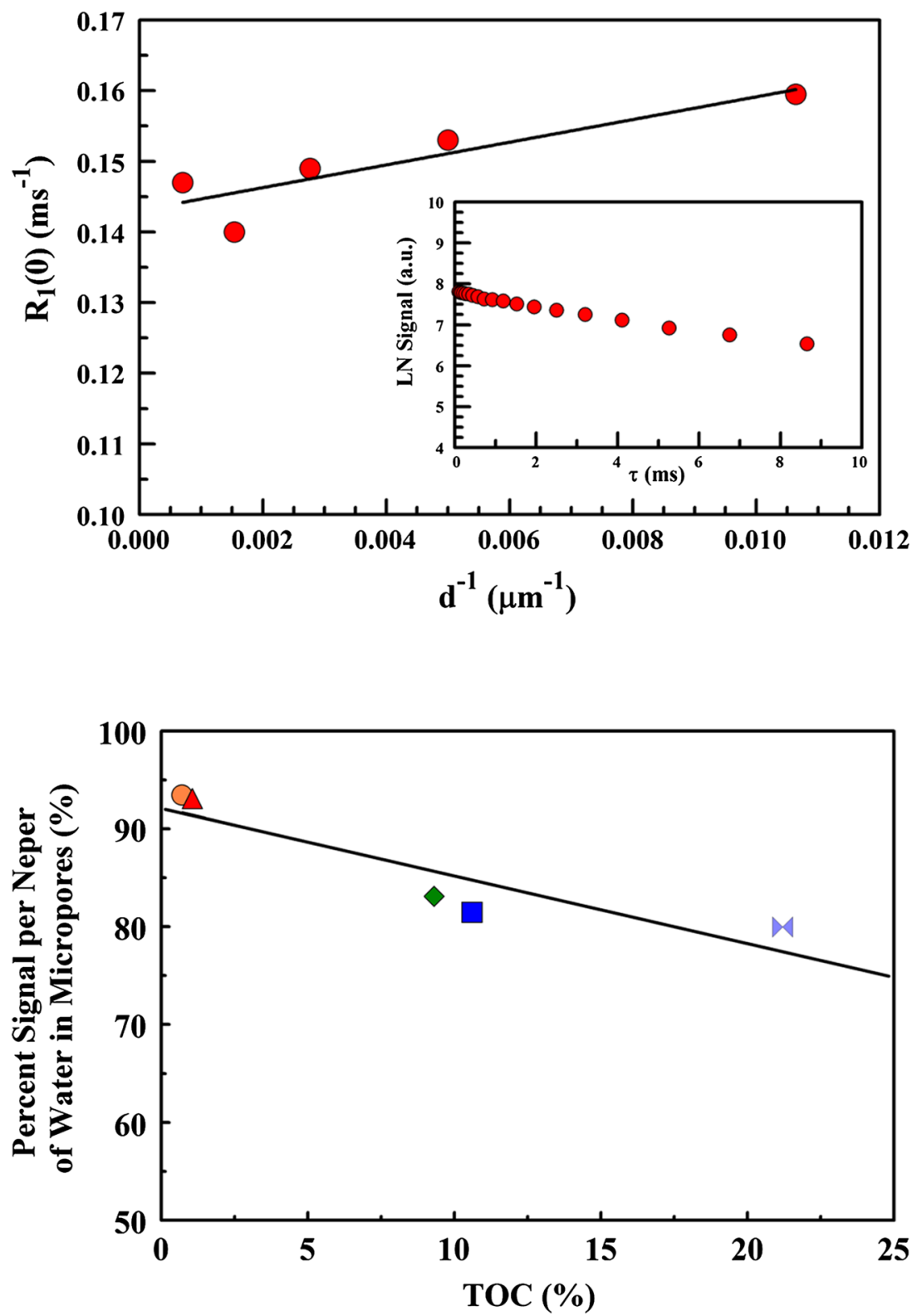

average pore size distribution ranging between $18 \mathrm{~nm}$ and $36 \mu \mathrm{m}$ has been obtained. Likewise, assuming an average cut-off value of $14 \mathrm{~ms}$ to delimitate the microporosity, the maximum dimension of the micropores is approximately set to $2.5 \mu \mathrm{m}$. This is reasonably comparable with the classification of Kodikara et al. (1999) that considers the range of micropores under $30 \mu \mathrm{m}$, including interparticle ( $1 \mu \mathrm{m} \div 3$ to $4 \mathrm{~nm}$ ) and intraparticle $(<3-4 \mathrm{~nm})$ range for clay structure. On the other side, the SSA of a particular sample measured by BET was equal to $28 \mathrm{~m}^{2} \mathrm{~g}^{-1}$, and from Eq. (2), using the volume of pore space $V$ of sample S2 (mass $=1 \mathrm{~g}$, volume of approximately $1 \mathrm{~cm}^{3}$ and porosity $=0.4$ ), the relaxivity obtained was equal to $\approx 1.5 \mu \mathrm{m} \mathrm{s}^{-1}$. This value is about little more than two orders of magnitude smaller than that obtained by NMR method, but, as described above, BET can underestimate the SSA, especially in presence of clay, which is generally present in all kind of soils. In order to implement the proposed technique, we do not need to know the exact amount of clay present in soil samples, so we did not measure it. Nevertheless, all kind of soils normally contains clay minerals, whose quantity can be measured in different ways. Here, we only observed the presence of clay by means of the gravimetric segregation of hydrated samples that, in the course of time, forms the typical soil layers. It is worthy to highlight that the proposed method overcomes the BET limitations. From BET analysis, a rough value of intra-grain porosity of 0.06 has been also 
estimated. This value corresponds to an average NMR relaxation time of $1.3 \mathrm{~ms}$, which sets the intra-grain pore size below $35 \mathrm{~nm}$.

\section{NMR Signal versus TOC and TOC/N}

The analysis of relaxation time distribution, using the above cut-off values, has allowed the identification of the signals of the sole water related to micropores. By repeating the procedures of sample measurement and data extraction, an average relative error of about $5 \%$ was estimated. Figure 3 shows the percentage of NMR signals of water in micropores versus the amount of TOC for each sample. It is possible to notice a trend that in first approximation can be assumed linear, in which an increase in the TOC leads to a decrease in detected water in micropores. A similar behavior appears in plotting the signal of micropores versus the $\mathrm{TOC} / \mathrm{N}$ ratio. This seems to indicate that the organic carbon tends to reduce the presence of water in micropores. This is in agreement with the results of Kirchmann and Gerzabek (1999) that indicate a correlation between the volume of soil micropores ranging between 1 and $5 \mu \mathrm{m}$ and the TOC content. Other studies report that the SSA of soils is reduced by organic coatings, suggesting that high TOC might reduce the surface area of the micropores (Mikutta et al. 2004). In the study of Wagai et al. (2009), concerning the extent and nature of OM coverage of soil mineral surfaces, it is reported that in sorption experiment, micropores are preferentially covered with OM with respect to other soil surfaces. These results could confirm some results of the present study, where the higher the TOC content, the less the spin-lattice relaxation signal from micropores, or equivalently the less water in micropores. It is noteworthy that the five investigated samples are intrinsically different samples, and part of the results is surely due to these intrinsic differences. Nevertheless, the observed trend seems significant, also taking in account these intrinsic differences.

\section{Conclusion}

At present, there are relatively few NMR relaxometry works on PMSSM samples and, in general, NMR $T_{1}$ relaxometry is a quite new approach in soil sciences. Moreover, finding adequate original core samples (not synthetic samples) from remediation fields, sample preparation and measurements is not yet a straightforward and established practice. The experimental procedures and measurement techniques described in this work have the advantage to generate sound and reproducible results. The $T_{1}$ characterization analysis compared with the measured TOC content seems to indicate that the higher the TOC content, the lower the presence of water in micropores, as shown by the correlation existing between the quantity of NMR signal of water in PMSSM micropores obtained from $T_{1}$ distributions and the TOC content. Although further studies will be necessary to implement a more robust application of NMR in this particular field of study, its application to mixtures of soils samples, and especially the $T_{1}$ characterization, suggests that NMR analysis could turn into a valuable tool to help a better understanding of the complexity of PMSSM, nevertheless disclosing implications in temporal characterization of soil degradation.

Acknowledgments The authors thank R. J. S. Brown for useful discussions and suggestions, Prof. Giuseppe Fornasari and Patricia Benito (Department of Industrial Chemistry, University of Bologna) for BET analysis, Laura Billi (ARPA Laboratories, Provincial Section, Ravenna), Barbara Ravaglia and Fausto Peddis (LAGIRN Laboratories, University of Bologna) for technical assistance.

\section{References}

Adani F (2004) I processi aerobici per il trattamento di rifiuti urbani. Quaderni di Ingegneria Ambientale. Cipa Srl, Milano

Assocarta (2012) Rapporto Ambientale dell'industria cartaria italiana (dati 2009-2010). Published in http://www.assocarta.it/it/ pubblicazioni.html

Bayer JV, Jaeger F, Schaumann GE (2010) Proton nuclear magnetic resonance (NMR) relaxometry in soil science applications. Open Magn Reson J 3:15-26

Beauchamp CJ, Charest MH, Gosselin A (2002) Examination of environmental quality of raw and composting de-inking paper sludge. Chemosphere 46:887-895

Blümich B (2003) NMR imaging of materials. Oxford University Press, USA

Bonoli A, Dall'Ara A (2012) A bioremediation case of ex-quarry area restored by paper sludge. J Biotechnol 157:499-504

Borgia GC, Bortolotti V, Brown RJS, Fantazzini P (1996) A robust method for calculating geometric mean times from multiexponential relaxation data, using only a few data points and only a few elementary operations. Magn Reson Imaging 14:895-897

Bortolotti V, Brown RJS, Fantazzini P (2009) UpenWin: a software to invert multi-exponential decay data. Distributed by University of Bologna, villiam.bortolotti@unibo.it

Bryar TR, Daughney CJ, Knight RJ (2000) Paramagnetic effects of Iron (III) species on nuclear magnetic relaxation of fluid protons in porous media. J Magn Reson 142:74-85

Coates GR, Xiao L, Prammer MG (1999) NMR logging-principles and applications. Gulf Publishing Company, Halliburton Energy Services Publication, Houston, TX

De Jonge H, Mittelmeijer-Hazeleger MC (1996) Adsorption of $\mathrm{CO}_{2}$ and $\mathrm{N}_{2}$ on soil organic matter: nature of porosity, surface area, and diffusion mechanisms. Environ Sci Technol 30(2):408-413

Dunn KJ, Bergman DJ, LaTorraca GA (2002) Nuclear magnetic resonance petrophysical and logging applications. In: Helbig K, Treitel S (eds) Handbook of geophysical exploration, seismic exploration. vol 32, Pergamon Press, London, p 583

EWC (European Waste Catalogue and Hazardous Waste List) (2002) Valid from 1 January 2002, Environmental Protection Agency

Fierro A, Norrie J, Gosselin A, Beauchamp CJ (1997) Deinking sludge influences biomass, nitrogen and phosphorus status of several grass and legume species. Can J Soil Sci 77:693-702 
Gombia M, Sykora S, Bortolotti V, Vacchelli E, Fantazzini P (2008) In: A numerical method to optimize presaturation sequences on multi-exponential samples, 9th international bologna conference, Cambridge, 13-17, July 2008

Helm L (2006) Relaxivity in paramagnetic systems: theory and mechanisms. Prog Nucl Magn Reson Spectrosc 49(1):45-64

Hürlimann MD, Latour LL, Sotak CH (1994) Diffusion measurements in sandstone core: nMR determination of surface-tovolume ratio and surface relaxivity. Magn Reson Imaging $12: 325-327$

Jackson MJ, Line MA (1997) Organic composition of a pulp and paper mill sludge determined by FTIR, 13C CP MAS NMR, and chemical extraction techniques. J Agric Food Chem 45: 2354-2358

Kirchmann H, Gerzabek MH (1999) Relationship between soil organic matter and micropores in a long-term experiment at Ultuna, Sweden. J Plant Nutr Soil Sci 162:493-498

Kleinberg RL (1999) Nuclear magnetic resonance. In: Wong P-Z (ed) Experimental methods in the physical sciences. vol 35, chap 9, Academic Press, San-Diego, CA, USA, pp 337-385

Kodikara J, Barbour SL, Fredlund DG (1999) Changes in clay structure and behavior due to wetting and drying. In: Proceedings of the 8th Australian-New Zealand Conference on Geomechanics. Hobart, pp 179-186

MD (Ministerial Decree), 5th Feb 1998, Environment minister, Italy, edited by Gazzetta Ufficiale della Repubblica Italiana, N 88, 16th April 1998

MD (Ministerial Decree), 13th Sept 1999, Agriculture minister, Italy, edited by Gazzetta Ufficiale della Repubblica Italiana, N 248, 21th Nov 1999

Mikutta C, Lang F, Kaupenjohann M (2004) Soil organic matter clogs mineral pores: evidence from ${ }^{1} \mathrm{H}-\mathrm{NMR}$ and $\mathrm{N}_{2}$ adsorption. Soil Sci Soc Am J 68:1853-1862
Mitreiter I, Oswald SE, Stallmach F (2010) Investigation of Iron (III)release in the pore water of natural sands by NMR relaxometry. Open Magn Reson J 3:46-51

Pennell K, Abriola L, Boyd S (1995) Surface area of soil organic matter reexamined. Soil Sci Soc Am J 59:1012-1018

Pignatello JJ (1998) Soil organic matter as nanoporous sorbent of organic pollutant. Adv Colloid Interface Sci 76-77:445-467

Provencher SW (1982) CONTIN: a general purpose constrained regularization program for inverting noisy linear algebraic and integral equations. Comput Phys Commun 27(3):229-242

Stevenson FJ (1994) Organic matter in soils: pools, distribution, transformations, and function. In Humus chemistry: genesis, composition, reactions, pp 1-23, 2nd edn. Wiley and sons editors, New York

Usherson J (1992) Recycled paper and sludge. Resour Recycl 95-100

Vannini M, Bonoli A, Bortolotti V, Macini P, Mesini E (2012) In: Natural Soil Mixed With Paper Mill Sludge Characterization By ${ }^{1} \mathrm{H}$ Nuclear Magnetic Resonance Longitudinal Relaxation Time. Proceedings of the 3rd congress on hazardous and waste materials, 12-14 Sept 2012

Vogt C, Galvosas P, Klitzsch N, Stallmach F (2002) Self-diffusion studies of pore fluids in unconsolidated sediments by PFG NMR. J Appl Geophys 50(4):455-467

Wagai R, Mayer LM, Kitayama K (2009) Extent and nature of organic coverage of soil mineral surfaces assessed by a gas sorption approach. Geoderma 149(1-2):152-160

Zdravkov BD, Cermák JJ, Šefara M, Janků J (2007) Pore classification in the characterization of porous materials: a perspective. Cent Eur J Chem 5(2):385-395 